\title{
Lattice gases in slab geometries
}

\author{
R. Brito* and M. H. Ernst ${ }^{\dagger}$ \\ University of Florida, Department of Physics, Gainesville, Florida 32611-2085
}

(Received 10 June 1991)

\begin{abstract}
Non-mean-field-type excess correlations at short times are present in three-dimensional (3D) computer simulations of the velocity autocorrelation function, but absent in $1 \mathrm{D}, 2 \mathrm{D}$, and $4 \mathrm{D}$. They are caused by ring collisions in a quasi-3D slab of size $2 \times L \times L \times L$ in a face-centered-hypercubic lattice with periodic boundary conditions, which is the only available lattice-gas cellular automaton with $3 \mathrm{D}$ isotropic fluid flow. We evaluate this excess correlation. The simulation data agree very well with our exact result.
\end{abstract}

PACS number(s): 05.20.Dd, 51.10.+y, 05.60.+w

In lattice-gas fluids there exist short-time correlations, which are apparently only present in three-dimensional (3D) systems, but absent in one, two, and four dimensions. It is the purpose of this paper to give a quantitative explanation of these 3D correlations, observed by van der Hoef and Frenkel in their computer simulations [1]. They found that the velocity autocorrelation function (VACF) of a tagged particle in a 3D lattice-gas cellular automaton (LGCA) deviates from the mean-field or Boltzmann results already after two time steps. This is not the case in one, two, and four dimensions $[2,3]$.

In fact, one can show that mean-field theory is still exact after two time steps for any LGCA, as long as it is contained in a volume with linear dimensions that exceed two lattice spacings in any of the $d$-independent space directions [4]. The occurrence of dynamically correlated ring collisions (that are responsible for the logarithmic density dependence of transport coefficients and for the long-time tails of current correlation functions), require at least three time steps.

How can the observed excess correlations be reconciled with the above statements? The fundamental reason is that there does not exist a regular 3D lattice with sufficient symmetry such that fourth-rank tensors, e.g., viscosity, are isotropic and such that the macroscopic fluid dynamic equations of the LGCA have the symmetry of an isotropic fluid $[5,6]$.

To recover isotropy in $3 \mathrm{D}$ LGCA's one actually considers a quasi-3D slab with $2 V=2 \times L \times L \times L$ sites, embedded in a $4 \mathrm{D}$ simple cubic lattice and only two lattice spacings wide in the fourth unphysical dimension. Only even sites $\mathbf{r}$ (with $r_{x}+r_{y}+r_{z}+r_{t}=$ even) are accessible. Hence the name face-centered-hypercubic (FCHC) model. Thus the number of available sites is $V=L^{3}$. There are $b=24$ allowed velocity states $\left\{\mathbf{c}_{i}\right\}(i=1, \ldots, b)$ per site: $( \pm 1, \pm 1,0,0),( \pm 1,0, \pm 1,0),( \pm 1,0,0, \pm 1),(0, \pm 1, \pm 1,0)$, $(0, \pm 1,0, \pm 1),(0,0, \pm 1, \pm 1)$.

The peculiar non-mean-field-type velocity correlations after two time steps are in fact a finite-size effect $[1,4]$ related to the slab geometry of the system's volume with a width of two lattice spacings in the fourth unphysical dimension. These correlations are therefore of geometric rather than dynamic origin. For similar reasons one would find excess correlations in a quasi-2D $2 \times 2 \times L \times L$ cell of the FCHC model or in a quasi-1D strip of width 2 constructed from the Frisch-Hasslacher-Pomeau (FHP) [7] model on the triangular lattice.

These geometric correlations at time $t=2$ exist of course in all LGCA's for all time correlation functions, whenever there is a macroscopic periodicity cell with at least one spatial period equal to 2 . Accurate computer simulation data are, however, only available for the VACF $\phi_{x}(t)=\left\langle v_{x}(0) v_{x}(t)\right\rangle$ of a single tagged particle in the FCHC model [1], for which we will carry out the explicit calculations.

Let $n_{i}(\mathbf{r}, t)$ and $\nu_{i}(\mathbf{r}, t)$ be the occupation numbers of a fluid particle and of a tagged particle at site $\mathbf{r}=$ $\left(r_{x}, r_{y}, r_{z}, r_{t}\right)$ with velocity $\mathbf{c}_{i}(i=1,2, \ldots, b)$. Then $\left\langle n_{i}(\mathbf{r}, t)\right\rangle=\rho / b=f$ is the fraction of fluid particles per velocity channel in equilibrium, and $\left\langle\nu_{i}(\mathbf{r}, t)\right\rangle=\langle\nu\rangle=$ $(b V)^{-1}$ that of the tagged particles.

The forward time evolution equation for $\nu_{i}(\mathbf{r}, t)(t>0)$ is given by

$$
S_{i} \nu_{i}(\mathbf{r}, t+1)=\nu_{i}(\mathbf{r}, t)+I_{i}(\nu(t), n(t)) .
$$

The fluid occupation number $n_{i}(\mathbf{r}, t)$ obeys a similar equation [8]. The streaming operator is defined through $S_{i} \nu_{i}(\mathbf{r})=\nu_{i}\left(\mathbf{r}+\mathbf{c}_{i}\right)$. The collision operator $I_{i}(\nu, n)$ is linear in $\nu$ and of degree $(b-1)$ in the $n$ 's. It depends on the fluid and tagged particle collision rules. Its fluctuation expansion with $n_{i}(\mathbf{r}, t)=f+\delta n_{i}(\mathbf{r}, t)$ has the form

$$
I_{i}(\nu, n)=\Omega_{i j} \nu_{j}+\Omega_{i j k} \nu_{j} \delta n_{k}+\cdots,
$$

where the coefficients $\Omega_{i j k l \ldots}$ are vanishing if two labels, out of the set $\{j k l \ldots\}$, are equal. The VACF at time $t$, i.e.,

$$
\phi_{x}(t)=V \sum_{\mathbf{r}, i, j} c_{i x} c_{j x}\left\langle\nu_{i}(\mathbf{r}, t) \nu_{j}(\mathbf{0}, 0)\right\rangle,
$$

can be calculated by iterating Eq. (1) $t$ times. For $t=1$ a combination of Eqs. (1)-(3) gives in matrix notation

$$
\phi_{x}(t)=b^{-1} c_{x}(\mathbb{1}+\Omega)^{t} c_{x} \equiv \phi_{0}(t),
$$


where $\Omega_{i j}$ is a $b \times b$ matrix and $c_{j x}$ is a $b$ vector. We further used the relation $\left\langle\nu_{i}(\mathbf{r}, 0) \nu_{j}\left(\mathbf{r}^{\prime}, 0\right)\right\rangle=\langle\nu\rangle \delta\left(\mathbf{r}, \mathbf{r}^{\prime}\right) \delta_{i j}$. After a single time step the exact result $\phi_{x}(1)$ equals the mean-field or Boltzmann result $\phi_{0}(1)$. To obtain $\phi_{x}(2)$ one might iterate Eqs. (1) and (2) twice, which yields a huge amount of terms, on the order of $b^{b}$. However, there exists a simpler method that yields about $b^{2}$ terms and uses the stationarity condition $\left\langle v_{x}(2) v_{x}(0)\right\rangle=$ $\left\langle v_{x}(1) v_{x}(-1)\right\rangle$. It allows us to calculate $v_{x}(1)$ from the forward evolution Eq. (1) and $v_{x}(-1)$ from the backward evolution equation [4] $(t>0)$

$\nu_{i}(\mathbf{r},-t-1)=S_{i} \nu_{i}(\mathbf{r},-t)+I_{i}(S \nu(-t), S n(-t))$,

where $(S n)_{i}=S_{i} n_{i}$. After some algebra we find $\phi_{x}(t)=$ $\phi_{0}(t)+\delta \phi_{x}(t)$ with the exact result for the excess correlation,

$$
\delta \phi_{x}(2)=b^{-1} f(1-f) \sum_{k, l} A_{x, k l}^{2} \delta\left(\mathbf{c}_{k}, \mathbf{c}_{l}\right)
$$

where $\mathbf{A}_{k l} \equiv \sum_{i} \mathbf{c}_{i} \Omega_{i k l}$ vanishes for $k=l$. As the Kronecker $\delta$ in Eq. (6) requires that $\mathbf{c}_{k}=\mathbf{c}_{l}$, the excess correlation $\delta \phi_{x}(2)$ vanishes except in slab or strip geometries, due to periodic boundary conditions (see Fig. 1). In such geometries the system has a macroscopic period of two lattice spacings, $\mathbf{L} \equiv \mathbf{c}_{a}-\mathbf{c}_{b}$, in at least one space direction, and the excess correlation receives a nonvanishing contribution from all pairs $(k l)$ in Eq. (6) that satisfy the equation $\mathbf{c}_{k}=\mathbf{c}_{l} \pm\left(\mathbf{c}_{a}-\mathbf{c}_{b}\right)$. In a quasi-1D strip on a triangular lattice there are four different pairs. In the quasi-3D slab on the FCHC lattice there are 12 different pairs, only four of which give a nonvanishing contribution. In concluding this part, we stress that the excess correlations (6) are not of dynamic, but of purely geometric origin.

In the second part of this paper the excess correlations will be evaluated for the FCHC model, and compared with the computer simulations [1]. To what extent does the result (6) depend on the details of the LGCA fluid and the tagged particle dynamics? In the standard LGCA's for nonequilibrium fluids (with Fermi exclusion) the identity of the fluid particle is lost during collision. To study the VACF of a tagged particle one needs to introduce additional collision rules for the collision between a tagged and a fluid particle, obeying the requirement that a tag-blind observer only sees the dynamics of the underlying fluid. Consequently, the exclusion principle also applies between a tagged and a fluid particle. There are many choices possible and we refer to Ref. [9] for a more elaborated discussion on tagged particle dynamics. Here we only consider the so-called maximally random collision rules, introduced by Frenkel and co-workers $[10,1]$.

Consider a possible multiple collision of fluid particles, where the set of input velocities $[p]=\left\{\mathbf{c}_{1}, \mathbf{c}_{2}, \ldots, \mathbf{c}_{p}\right\}$, chosen out of $[b]$, scatters (with a certain probability) into the output velocities $\left[p^{*}\right]=\left\{\mathbf{c}_{2}{ }^{*}, \mathbf{c}_{2}{ }^{*}, \ldots, \mathbf{c}_{p}{ }^{*}\right\}$. If the input set $[p]$ contains the tag, and the maximally random collision rules apply, then the tag will be with equal probability on any of the outgoing particles. The collision term for this $[p]$-tuple collision is

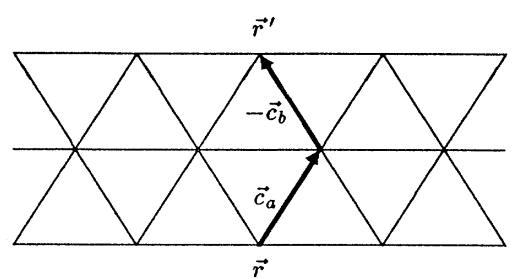

FIG. 1. Quasi-1D strip of width 2 with periodic boundary conditions $\mathbf{r}^{\prime}=\mathbf{r}+\mathbf{c}_{a}-\mathbf{c}_{b}$.

$$
\begin{aligned}
I_{i}^{(p)}(\nu, n)= & -\nu_{i} n([p]-i) \bar{n}([b]-[p]) \\
& +\frac{1}{p} \sum_{j \in\left[p^{*}\right]} \nu_{j}^{*} n\left(\left[p^{*}\right]-j^{*}\right) \bar{n}\left([b]-\left[p^{*}\right]\right),
\end{aligned}
$$

where $n([p])=n_{i_{1}} n_{i_{2}} \ldots n_{i_{p}}$ and $\bar{n}([p])=\left(1-n_{i_{1}}\right)(1-$ $\left.n_{i_{2}}\right) \ldots\left(1-n_{i_{p}}\right)$.

This schematic formula for the maximally random collision rules, combined with momentum conservation, is sufficient to show that $\sum_{i} \mathbf{c}_{i} I_{i}(\nu, n) \equiv \mathbf{A}(\nu, n)$ is independent of the collision rules of the underlying LGCA fluid and is given by

$$
\mathbf{A}(\nu, n)=\sum_{p=2}^{b} \sum_{[p] \subset[b]} \sum_{i=1}^{p} \underbrace{}_{\substack{p=1 \\ \times \nu_{i} n([p]-i) \bar{n}([b]-[p]) .}}
$$

By expanding $\mathbf{A}(\nu, n)=\mathbf{A}_{k} \nu_{k}+\mathbf{A}_{k l} \nu_{k} \delta n_{l}+\cdots$ in powers of the fluctuation $\delta n=n-f$ around equilibrium, we find, after a lengthy but straightforward calculation,

$$
\begin{aligned}
& \mathbf{A}_{k}=-\lambda \mathbf{c}_{k} \\
& \mathbf{A}_{k l}=\frac{1}{2}\left(\mathbf{c}_{k}+\mathbf{c}_{l}\right) g+\frac{1}{2}\left(\mathbf{c}_{k}-\mathbf{c}_{l}\right) h
\end{aligned}
$$

and $\mathbf{A}_{k k}=0$ because $\Omega_{i k k}$ vanishes. All three coefficients $\lambda, g$, and $h$ are functions of the reduced density $f=\rho / b$ and of the bit number $b$ :

$$
\begin{aligned}
\lambda(f, b)=- & {[(b-1) f]^{-1}\left[1-b f-(1-f)^{b}\right] } \\
g(f, b)=- & {\left[(b-1)(b-2) f^{2}\right]^{-1} } \\
\times & \times\left[2-b f-b(1-f)^{b-1}+(b-2)(1-f)^{b}\right]
\end{aligned}
$$

$h(f, b)=-[(b-1) f]^{-1}\left[1-(1-f)^{b-1}\right]$.

The coefficient $\lambda$ is in fact an eigenvalue of $\Omega$ [9], i.e., $\Omega_{i j} \mathbf{c}_{j}=-\lambda \mathbf{c}_{i}$. Consequently the mean-field approximation in Eq. (4) becomes

$$
\phi_{0}(t)=\phi_{x}(0)(1-\lambda)^{t} .
$$

The VACF is normalized as $\phi_{x}(0)=\left\langle v_{x}^{2}(0)\right\rangle=c_{0}^{2}$. Here the sound velocity $c_{0}=1 / \sqrt{2}$ for the FCHC model and $c_{0}=\sqrt{3 / b}$ for the triangular lattice, where $b=7$ with and $b=6$ without rest particle. Combination of (6), (9), and (10) gives the exact result for the excess correlation in the quasi-3D FCHC model $(b=24)$ induced by the 
slab geometry:

$\delta \phi_{\alpha}(2)= \begin{cases}\frac{1}{6} f(1-f)[g(f, 24)]^{2} & (\alpha=x, y, z) \\ \frac{1}{2} f(1-f)[h(f, 24)]^{2} & (\alpha=t) .\end{cases}$

For a quasi-1D strip on a triangular lattice with six moving particles $(b=6)$ or with one additional rest particle $(b=7)$ we obtain

$$
\begin{aligned}
& \delta \phi_{x}(2)=\frac{1}{b} f(1-f)[g(f, b)]^{2}, \\
& \delta \phi_{y}(2)=\frac{3}{b} f(1-f)[h(f, b)]^{2} .
\end{aligned}
$$

Details are planned to be published elsewhere [14].

We conclude with a number of comments.

(1) In Fig. 2 the simulation data for the excess correlation in the VACF of the quasi-3D FCHC model at $t=2$ are compared with our exact result. The abscissa shows the normalized excess $\Delta(t)=\left[\phi_{x}(t)-\phi_{0}(t)\right] / \phi_{x}(0)=$ $\delta \phi_{x}(t) / c_{0}^{2}$ after two time steps as a function of the reduced density $f$ (solid line). The circles [1] and squares (which represent low-density simulations at $f=0.1$ and 0.2 of very high statistical accuracy [11]) show very good agreement with the exact result $(12 \mathrm{a})$.

The dashed line in Fig. 2 shows the mean-field result $\phi_{0}(t) / \phi_{x}(0)=(1-\lambda)^{t}$ at $t=2$. For low densities $(f \lesssim$ $0.4)$ the mean-field result $\phi_{0}(2) \gg \delta \phi_{x}(2)$; for $0.5<f<$ 0.9 both contributions to $\phi_{x}(2)$ are of the same order. At high densities $(f \gtrsim 0.95) \delta \phi_{x}(2)$ exceeds $\phi_{0}(2)$ by more than a factor of 10 . In all LGCA's without slab geometry, i.e., with all macroscopic periods larger than 2, the VACF is exactly given by the mean-field result $\phi_{x}(2)=\phi_{0}(2)$, which has been verified by extensive simulations for the dimensionalities $d=1,2$ and $4[2,3,11]$.

(2) Equation (12b) gives the VACF $\phi_{\alpha}(2)=$ $\left\langle v_{\alpha}(2) v_{\alpha}(0)\right\rangle=\phi_{0}(2)+\delta \phi_{\alpha}(2)$ for the fourth unphysical dimension (labeled $\alpha=t$ ). It differs from the VACF in the three physical directions $(\alpha=x, y, z)$. The slab geometry of the quasi-3D FCHC model breaks the 4D isotropy of the second rank tensor $\left\langle v_{\alpha}(2) v_{\beta}(0)\right\rangle$ with $(\alpha, \beta)=\{x, y, z, t\}$. As a $3 \mathrm{D}$ object the tensor is still symmetric.

As a consequence of the slab geometry the unphysical diffusivity $D_{\alpha}=\sum_{t=0}^{\infty} \phi_{\alpha}(t)-\frac{1}{2} \phi_{\alpha}(0)$ for $\alpha=t$ is different from the physical coefficient of self-diffusion $D_{x}=D_{y}=D_{z}$, as soon as non-mean-field terms are taken into account in the theory. Similar comments apply to the quasi-1D VACF $\phi_{x}(2)$ in Eq. (13), where $\phi_{y}(2)$ corresponds to the unphysical direction.

In conclusion, the slab geometry, introduced in $3 \mathrm{D}$ LGCA fluids to recover the isotropy of the Navier-Stokes equation, leads to spurious (rather small) contributions to time correlation functions and transport coefficients. These spurious contributions are in fact "finite-size effects," induced by the small width of two lattice spacings in the fourth unphysical spatial direction.

(3) In our analysis of geometric correlations we have only considered the contributions in Eq. (2), proportional to $\nu \delta n$. The higher-order terms have been analyzed in a similar manner. In systems with a slab geometry, having

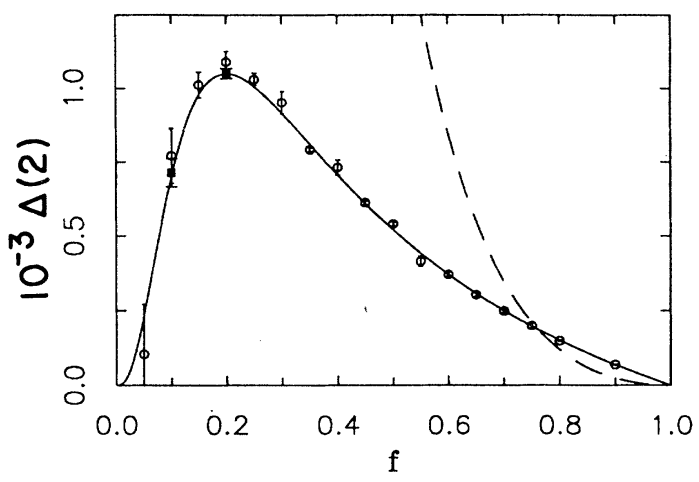

FIG. 2. The excess (solid line) $\Delta(t)=\left[\phi_{x}(t)-\right.$ $\left.\phi_{0}(t)\right] / \phi_{x}(0)$ in the VACF $\phi_{x}(t)=\left\langle v_{x}(0) v_{x}(t)\right\rangle$ at time $t=2$ over the Boltzmann value $\phi_{0}(2) / \phi_{x}(0)=(1-\lambda)^{2}$ (dashed line) as a function of reduced density $f$. Open circles (o) are the simulation data of Ref. [1] and solid squares ( $\square$ ) at $f=0.1,0.2$ are data of higher statistical accuracy [11].

only a single macroscopic period equal to 2, all higherorder terms give a vanishing contribution. Examples of such systems are the quasi-3D FCHC model in a slab of size $2 \times L \times L \times L$ or in the quasi-1D FHP model in a strip of size $2 \times L$.

However, in slab geometries with two macroscopic spatial periods equal to 2 , also the term $\Omega_{i j k l} \nu_{j} \delta n_{k} \delta n_{l}$ gives a nonvanishing contribution to $\delta \phi_{\alpha}(2)$, but not the higherorder terms. An example of such a system is the quasi-2D FCHC model on a slab of size $2 \times 2 \times L \times L$ [14].

(4) Extensions of the present theory toward larger times involves a systematic extension of kinetic theory, which accounts for correlated collisions [4], that have been neglected in the Boltzmann approximation. The term calculated in Eq. (6) is in fact a contribution from the so-called ring collisions, requiring that two particles, say, $(k l)$ in Eq. (6), after a previous collision recollide again, expressed by $\delta\left(\mathbf{c}_{k}, \mathbf{c}_{l}\right)$. In systems with slab geometry, having spatial period 2, and obeying periodic boundary conditions, the ring (closed-polygon) condition can be satisfied within two time steps, yielding a nonvanishing contribution. In systems large in all spatial directions, the ring collisions give only nonvanishing contributions after at least three time steps. We have also obtained $[4,14]$ an approximate ring kinetic equation tor the VACF. It predicts the excess correlation $\delta \phi_{x}(t)=\phi_{x}(t)-\phi_{0}(t)$ for all times. This approximation is still exact for $t=2$. For $t=3$ it contains the typical recollision events (dynamical correlation) contributing in all geometries, as well as the additional geometric correlations, induced by slab geometries. For long times the approximate ring equation reduces to the mode coupling results $[2,10,12,13]$ that show good agreement with simulation data for the VACF $[1-3,12]$.

(5) For the maximally random tagged particle collision rules [see above Eq. (7)], the mean-field result $\phi_{0}(t)=\phi_{x}(0)(1-\lambda)^{t}$ as well as the geometric correlation $\delta \phi_{\alpha}(2)$ are totally independent of the collision rules of the underlying LGCA fluid. Even in case all collisions 
between fluid particles would be replaced by noninteracting ones, but the tag is still redistributed over the outgoing states according to the maximally random collision rules, the Boltzmann result for the VACF $\phi_{0}(t)$, and for the diffusion coefficient as well as the geometric correlation $\delta \phi_{x}(2)$, remain unaltered. The proof is based on momentum conservation. For the diffusion coefficient and the VACF this has been observed already in Ref. [9]. The dynamic ring correlations in $\delta \phi_{\alpha}(3)$ will however depend on the collision rules of the LGCA fluid. Also, for different tagged particle collision rules and for fluid type correlation functions, such as the stress-stress correlation, the excess geometric correlation in slab geometries after two time steps does depend explicitly on the collision rules of the LGCA fluid.

It is a pleasure to thank J. W. Dufty, D. Frenkel, and M. van der Hoef for many stimulating and clarifying discussions, and in particular D.F. and M.v.d.H. for providing us with some unpublished simulation data. R.B. acknowledges support from a DGICYT Project No. PB880140 and M.H.E. from a NATO Collaborative Research Grant.
* Permanent address: Facultad de Ciencias Físicas, Universidad Complutense, 28040 Madrid, Spain.

$\dagger$ Permanent address: Institute for Theoretical Physics, University of Utrecht, P.O. Box 80006,3508 TA Utrecht, The Netherlands.

[1] M.A. van der Hoef, and D. Frenkel, Physica D 47, 191 (1991).

[2] T. Naitoh, M.H. Ernst, M. van der Hoef, and D. Frenkel, Phys. Rev. A 44, 2484 (1991); unpublished.

[3] M.A. van der Hoef, M. Dijkstra, and D. Frenkel, Europhys. Lett. (to be published).

[4] T.R. Kirkpatrick and M.H. Ernst (unpublished).

[5] S. Wolfram, J. Stat. Phys. 45, 471 (1986).

[6] D. d'Humières, P. Lallemand, and U. Frisch, Europhys. Lett. 2, 291 (1986).
[7] U. Frisch, B. Hasslacher, and Y. Pomeau, Phys. Rev. Lett. 56, 1505 (1986).

[8] U. Frisch, D. d'Humières, B. Hasslacher, P. Lallemand, Y. Pomeau, and J.P. Rivet, Complex Systems 1, 649 (1987).

[9] M.H. Ernst and T. Naitoh, J. Phys. A 24, 2555 (1991).

[10] D. Frenkel and M.H. Ernst, Phys. Rev. Lett. 63, 2165 (1989).

[11] M. van der Hoef and D. Frenkel (private communication).

[12] M. van der Hoef and D. Frenkel, Phys. Rev. A 41, 4277 (1990).

[13] T. Naitoh, M.H. Ernst, and J.W. Dufty, Phys. Rev. A 42, 7187 (1990).

[14] R. Brito and M.H. Ernst (unpublished). 